\section{NP41 Year 4 of the Children's Healthy Living Program for Remote Underserved Minority Populations of the Pacific Region (CHL)}

Rachel Novotny, PhD RDN LD, novotny@hawaii.edu, University of Hawaii at Manoa, 1955 East West Road, Agricultural Science Building 216, Honolulu, HI 96822; A. Areta, MA, American Samoa Community College; A. Bersamin, PhD, University of Alaska at Fairbanks; J. Deenik, PhD, University of Hawaii at Manoa; J. Kim, PhD, Northern Marianas College; R. L. Guerrero, PhD, RDN

Objective: Prevent child obesity in the Pacific through capacity building.

Description: US-Affiliated Pacific Land Grant Colleges and local partners implemented a child obesity prevalence survey and a randomized, controlled environmental intervention supporting community agency actions and role models. Obesity prevention degree-providing scholarship and training program is being provided to 21 recipients. Graduates and successful activities are being integrated into regional infrastructures.

Evaluation: Pacific-wide data systems monitor and evaluate program activities and health outcomes, providing data for outreach and policy development.

Conclusions and Implications: Alignment of CHL program actions at multiple levels and sectors is vital for sustainable childhood obesity prevention.

Funding: USDA Grant \#2011-68001-30335.

\section{NP42 Food Weight Estimation: A Comparative Analysis of Digital Food Imaging Analysis and 24-Hour Dietary Recall}

Roberto Trevño, MD, rtrevino@sahrc.org, Social \& Health Research Center, 1302 So St Mary's Street, San Antonio, TX 78210; A. Ravelo, MS, Florida International University; E. Birkenfeld, BS, CHES, Social \& Health Research Center; M. Murad, BS, University of Incarnate Word; J. Diaz, BS RD

Objective: To improve accuracy in food weight estimation using the Digital Food Imaging Analysis (DFIA). The DFIA uses digital photography, optical recognition software, and the USDA Standard Reference database to identify food and calculate nutritional value. Photographs of meals are taken before and after to calculate consumption. In this abstract food weight estimation of DFIA was compared to 24-hour dietary recall (24HDR).

Description: Food weight is the fundamental unit for nutrient analysis. The challenge in assessing dietary intake is collecting accurate food volumes and converting these to food weight. The 24-HDR uses memory recalls supported by visual guides (e.g. deck of cards, baseball, food pictures and models) to estimate food volume and then convert to weight. As a result, the estimation of food weight is susceptible to measurement errors. Because of these limitations, the DFIA technique was developed to improve accuracy in estimating food weight. A total of 120 students (ages 8-9 years) from two schools with parent consent and complete data were included in this analysis ( $n=120$ meals; 57 breakfast +63 lunch). A 24-HDR was collected the following day and DFIA was collected the same day of the analysis. Food weight for 24-HDR was estimated by Nutrition Data System for Research software (NDSR; version 4.04), and for DFIA it was estimated by demarcating surface area of food items in photographs before and after consumption, and converted to weights using image analysis properties. For reference, meals were weighed with an electronic scale at the school cafeteria cash register and again at the disposal window to calculate food weight consumption. Validity was examined by comparing real food weight and the two dietary intake instruments.

Evaluation: A comparison between the estimated food weight by NDSR and DFIA to real food weight was done. The regression model shows that DFIA had a better fit than NDSR with $\mathrm{R}$ square $=0.351>0.088$, respectively. The Pearson's correlation also displayed a stronger correlation between DFIA and real weight (0.592) than NDSR and real weight (0.297). Lastly the Intraclass Correlation Coefficient for single measures showed that DFIA had a reliability of 59\% compared with $30 \%$ for NDSR; and for average measures DFIA had a reliability of $74 \%$ compared to $46 \%$ for NDSR. These findings support that the DFIA is more accurate than NDSR to estimate food weights.

Conclusions and Implications: The DFIA showed to be more accurate estimating food weight. The measurement results are closer to the real values, because it negates the confounding errors associated with food weight conversion using food models or memory recall.

Funding: USDA Grant \#2011: 67001-30071.

\section{NP43 Healthy Caregivers-Healthy Children: Two Year Follow-Up Findings}

Ruby Natale,PhD, PSyD, rnatale@med.miami.edu, University of Miami School of Medicine/Mailman Center for Child Development, 1601 NW 12th Avenue, Room 4010, Miami, FL 33136; S. Messiah, PhD, MPH; A. Delamater, PhD; S. Uhlhorn, PhD

Objective: To assess the impact of an early childhood obesity prevention intervention on dietary patterns and BMI over two school years.

Description: A total of 28 centers were randomly assigned to: (1) Control Arm (safety intervention) or (2) Intervention Arm that received a multicomponent nutrition and physical activity program.

Evaluation: Data was collected at baseline, 6-months, 12-months, and 24-months post intervention. Analyses found children in the treatment group had a negative slope (beta $=-1.95$, se $=0.97, p=0.04$ ), indicating less increase in BMI percentile versus control children.

Conclusions and Implications: After two years of a preschool obesity prevention intervention, results indicate the potential long term impact of this program.

Funding: USDA Grant \#2009-05065. 